\title{
ON FINITE HILBERT TRANSFORMS
}

\author{
BY \\ KEVIN F. CLANCEY $\left({ }^{1}\right)$
}

ABSTRACT . Let $E$ be a bounded measurable subset of the real line. The finite Hilbert transform is the operator $H_{E}$ defined on one of the spaces $L^{p}(E)$ $(1<p<\infty)$ by

$$
H_{E} f(x)=(\pi i)^{-1} \int_{E} f(t)(t-x)^{-1} d t
$$

here, the singular integral is interpreted as a Cauchy principal value. The main result establishes that for $H_{E}$ to be Fredholm on $L^{p}(E)$, when $p \neq 2$, it is necessary and sufficient that $E$ be equal almost everywhere to a finite union of intervals. The sufficiency of this condition was established in $1960 \mathrm{by} \mathrm{H}$. Widom. In the case where $E$ is not a finite union of intervals and $p<2$ it is shown that the operator $H_{E}$ has an infinite dimensional null space. The method of proof is constructive.

Introduction. Let $E$ be a Lebesgue measurable nonnull subset of the real line $\mathbf{R}$. The notations $L^{p}(E) \quad(1 \leqslant p \leqslant \infty)$ will be used for the customary Lebesgue spaces on $E$. For $f$ in $L^{p}(\mathbf{R}), p \geqslant 1$, the Hilbert transform of $f$ at $x$ is defined by

$$
H f(x)=\lim _{\epsilon \rightarrow 0}(\pi i)^{-1} \int_{|t-x| \geq \epsilon} f(t)(t-x)^{-1} d t
$$

provided this latter limit exists. It is known that if $f$ is in $L^{p}(R)$, for $p \geqslant 1$, then $H f$ exists a.e. Moreover, when $p>1$, the map $f \rightarrow H f$ defines a bounded linear operator on $L^{p}(\mathbf{R})$ (see, e.g., Garsia [2]). Obviously, it is possible to consider the reduced Hilbert transform $H_{E}$ defined on $L^{p}(E)(p \geqslant 1)$ by $H_{E} f(x)=$ $(\pi i)^{-1} \int_{E} f(t)(t-x)^{-1} d t$; here, the integral is clearly singular and must be interpreted as the Cauchy principal value defined in equation (1). The map $f \rightarrow$ $H_{E} f$ is a bounded operator on $L^{p}(E)(p>1)$. If the set $E$ is bounded, then the operator $H_{E}$ will be referred to as a finite Hilbert transform.

Widom [8] described the spectra of the finite Hilbert transforms on all of the $L^{p}$ spaces $(1<p<\infty)$. In order to state Widom's result it is necessary to introduce the following notation: let $p>1$, define

Received by the editors August 26, 1974.

AMS (MOS) subject classifications (1970). Primary 45E05; Secondary 44A15.

(1) This work was supported by the National Science Foundation. 


$$
A_{p}=\{\lambda \in C: \arg [(\lambda+1) /(\lambda-1)]=2 \pi / p\} .
$$

Note that the branch of the argument is chosen such that $0 \leqslant \arg z<2 \pi, z \neq 0$. For each $p>1$, the set $A_{p}$ is a circular arc joining the points \pm 1 and passing through $-i \cot (\pi / p)$. If $p, q$ are conjugate indices so that $p^{-1}+q^{-1}=1$, then $A_{p}=-A_{q}$. For each $p>1$, the region bounded by $A_{p} \cup A_{q}\left(p^{-1}+q^{-1}=1\right)$ will be denoted by $R_{p}$. The set $R_{p}$ has the description

$$
R_{p}=\{\lambda \in \mathrm{C}: 2 \pi / q<\arg [(\lambda+1) /(\lambda-1)]<2 \pi / p\}, \quad p<2 .
$$

The results in the following theorem appear in Widom [8].

THEOREM. (a) Let $E$ be a bounded measurable subset of $\mathbf{R}$ and let $p>1$. The spectrum of the operator $H_{E}$ on $L^{p}(E)(p \neq 2)$ is the closure of $R_{p}$.

(b) Assume $E$ is a.e. the disjoint union of $m$ bounded intervals. In case $p$ $<2$, each $\lambda$ in $R_{p}$ is an eigenvalue of $H_{E}$ of multiplicity $m$ and the range of $H_{E}-$ $\lambda$ is closed. If $p>2$ and $\lambda$ is in $R_{p}$, then the operator $H_{E}-\lambda$ is one to one and the range is of codimension $m$.

Actually, Widom does not include a proof of the fact that $R_{p}$ is a subset of the spectrum of $H_{E}$ on $L^{p}(E)$ (for any bounded measurable subset of $\mathbf{R}$ ); however, this remark was added in proof $[8$, p. 157].

The book of Tricomi [7] contains many results on the finite Hilbert transform when $E=[0,1]$. Shamir [5] and more recently Juberg [4] obtained by other methods all of the results in the above theorem for the case $E=[0,1]$. There is a series of papers by Gohberg and Krupnik (see, e.g. [3] and the references cited) on singular integral operators acting on $L^{p}$-spaces with weights and having piecewise continuous coefficients. Gohberg and Krupnik obtain the results in part (b) of the above theorem for the analogue of the Hilbert transform acting on a union of smooth arcs in the complex plane.

The basic method used in this note to study singular integral equations is in Tricomi's book [7]. Similar methods were explored for other singular integral equations with measurable coefficients in [1]. The main result, which is established in $\S 2$, shows that if $E$ is bounded and not a finite union of intervals, then for $p<2$, each $\lambda \in R_{p}$ is an eigenvalue of $H_{E}$ of infinite multiplicity.

1. Preliminaries. This section contains some basic facts about the Hilbert transform and methods connected with singular integral equations which will be used in the following section to establish the main result.

The duality between the spaces $L^{p}(E)$ and $L^{q}(E)$ when $p^{-1}+q^{-1}=1$ is given by $\langle f, g\rangle=\int_{E} \bar{f} d t, f \in L^{p}(E)$ and $g \in L^{q}(E)$. If $p>1$, then the reduced Hilbert transform $H_{E}$ on $L^{q}(E)$ is the adjoint of $H_{E}$ on $L^{p}(E)$. 
Define for $\theta$ in $L^{p}(\mathbf{R}), p \geqslant 1$, the Cauchy transform of $\theta$ at $z$, where $\operatorname{Im} z$ $\neq 0$, by

$$
\Theta(z)=\frac{1}{2 \pi i} \int \frac{\theta(t)}{t-z} d t
$$

The function $\Theta$ is separately analytic in the upper and lower half-planes and the limits $\Theta^{ \pm}(x)=\lim _{y \downarrow 0} \Theta(x \pm i y)$ exist almost everywhere. Further, the functions $\Theta^{ \pm}$are connected to $H \theta$ by the well-known barrier identities

$$
\Theta^{+}-\Theta^{-}=\theta, \quad \Theta^{+}+\Theta^{-}=H \theta .
$$

See, e.g., Titchmarsh [6].

The Hardy spaces of the upper half-plane are defined in the following manner: let $p \geqslant 1$, the Hardy space $H^{p}$ consists of all functions $F$ analytic in the upper half-plane which satisfy

$$
\sup _{y>0} \int|F(x+i y)|^{p} d x<\infty .
$$

The space $H^{\infty}$ is the collection of bounded analytic functions in the upper halfplane. If $F$ is in $H^{p} \quad(1 \leqslant p \leqslant \infty)$, then $F^{+}(x)=\lim _{y+0} F(x+i y)$ exists a.e. and belongs to $L^{p}(\mathbf{R})$. The basic result about Hardy spaces which is needed in the sequel is the following: let $F$ belong to $H^{p}$ for some $p>1$, then $\operatorname{Re} F^{+}=$ $i H\left(\operatorname{Im} F^{+}\right.$) (see, e.g., Titchmarsh [6]).

The following lemma is an easy modification of a result which appears in Zygmund [10, p. 164].

LEMMA 1. Let $\theta$ be real valued essentially bounded with compact support. Suppose in some interval $(a, b)$ the function $\theta$ can be written in the form $\theta=u$. $+v+w$, where $u, v, w$ are real valued measurable functions such that (i) $u$ is continuous on $(a, b)$, (ii) for some $\epsilon>0$ and $p \geqslant 1,|v(x)| \leqslant \pi / 2 p-\epsilon$ a.e. on $(a, b)$, and (iii) $w$ is decreasing on $(a, b)$. Then $\exp |i H \theta|$ belongs to $L^{p}\left(a^{\prime}, b^{\prime}\right)$, for any closed subinterval $\left[a^{\prime}, b^{\prime}\right]$ of $(a, b)$.

It is not true that every function $F$ which is analytic in the upper halfplane for which $F^{+}(x)=\lim _{y \downarrow 0} F(x+i y)$ exists a.e. and belongs to $L^{p}(\mathbf{R})$ is automatically in $H^{p}$. The next lemma shows that for special types of analytic functions this latter statement is true. A proof of the following lemma is in [1].

LEMMA 2. Let $\theta$ be a real valued, essentially bounded and have compact support. Let $\Theta$ be the analytic function defined, for $\operatorname{Im} z>0$, by

$$
\Theta(z)=\exp \left[\frac{1}{\pi} \int \frac{\theta(t)}{t-z}\right]-1
$$


If the function $\Theta^{+}(x)=\lim _{y \downarrow 0} \Theta(x+i y)$ (which obviously exists a.e.) belongs to $L^{p}(\mathbf{R})$, for some $p>1$, then $\Theta$ belongs to $H^{p}$.

The limit function $\Theta^{+}$of the function $\Theta$ defined by (3) is

$$
\Theta^{+}=\exp [i(H \theta+\theta)]-1 \text {. }
$$

This follows easily from the barrier relations (2). The fact that $\theta$ has compact support permits one to use a power series argument to conclude that $\Theta^{+}$belongs to $L^{p}(\mathbf{R} \backslash h)$ for any $p \geqslant 1$, where $I$ is any open interval containing the support of $\theta$.

Further, if $\Theta^{+}$belongs to $L^{p}(\mathbf{R})$ (a decision which is aided by Lemma 1), then the preceding lemma implies $\Theta$ is in $H^{p}$ and consequently $\operatorname{Re} \Theta^{+}=$ $i H\left[\operatorname{Im} \Theta^{+}\right]$. This leads to the equation

$$
\cos \theta \exp (i H \theta)=i H[\sin \theta \exp (i H \theta)]+1 .
$$

It will be instructive to consider an example to show how equation (5) can be used to solve singular integral equations. Let $E$ be a bounded measurable subset of $\mathbf{R}$ and let $\theta$ be the function

$$
\theta(t)= \begin{cases}\pi / 2, & t \in E, \\ 0, & t \notin E .\end{cases}
$$

It follows from Lemma 1 that $\Theta^{+}=\exp [i(H \theta+\theta)]-1$ is in $L^{p}(\mathbf{R})$ for any $p<2$. Equation (5) then yields

$$
\frac{1}{\pi i} \int_{E} \frac{\exp (i H \theta(t))}{t-x} d t=-i, \quad x \in E .
$$

In other words, for any $p<2$, the function $f=\exp (i H \theta)$ is an $L^{p}(E)$ solution of the equation $H_{E} \phi=-i \chi_{E}$; where $\chi_{E}$ denotes the characteristic function of $E$. It is not hard to construct a second linearly independent $L^{p}(E)$ solution of $H_{E} \phi=-i \chi_{E}$ (just repeat the above argument with $\theta$ replaced by $\theta^{\prime}=-\pi \chi_{E} / 2$ ). This leads to an $L^{p}(E)$ solution of the homogeneous equation $H_{E} \phi=0$ for any $p<2$.

In the next section when $E$ is not a finite union of intervals the above method will be adapted to construct an infinite number of linearly independent $L^{p}(E)$ solutions of $H_{E} \phi=0$.

It will be nęcessary to know that the Hilbert transform of the characteristic function of a bounded measurable set is unbounded. More precisely, suppose $E$ is a bounded measurable subset of $\mathbf{R}$ and $(a, b)$ is any interval which intersects both $E$ and its complement $E^{c}$ in a set of positive measure, then the 
function $i H \chi_{E}$ is not bounded (essentially) from above or below on $(a, b) \cap E$ or $(a, b) \cap E^{c}$. This result follows easily from the results in Zygmund [9, p. 17].

2. The main result. Throughout this section it is assumed that $E$ is a bounded measurable subset of $\mathbf{R}$ which is not equal a.e. to a finite union of intervals. Set $m=$ ess $\inf _{t \in E} t$ and let $a, b$ be fixed real numbers such that $a<b<$ $m$. Since $E$ is not a finite union of intervals, it is easy to choose an infinite monotone sequence $\left\{\lambda_{n}\right\}_{n=0}^{\infty}$ such that both $E$ and $E^{c}$ intersect the intervals $I_{n}$ $(n=1,2, \ldots)$ in a set of positive measure; where $I_{n}$ is defined to be $\left(\lambda_{n-1}\right.$, $\left.\lambda_{n}\right)$ if $\left\{\lambda_{n}\right\}$ increases and $\left(\lambda_{n}, \lambda_{n-1}\right)$ if $\left\{\lambda_{n}\right\}$ decreases.

It will be assumed for the present that $p$ is a ixed number, $1<p<2$, and that $\lambda=i \mu$ where $\mu$ is a fixed real number so that $\alpha=\arg [(\lambda+1) /(\lambda-1)]$ satisfies $2 \pi q^{-1}<\alpha<2 \pi p^{-1}$. Fix $n \geqslant 1$ and define the sequence of functions $\left\{\theta_{j}^{n}\right\}_{j=1}^{\infty}$, where

$$
\theta_{j}^{n}(t)= \begin{cases}\alpha / 2, & t \in E \\ \pi / 2, & t \in(a-1 / j, a) \\ \pi, & t \in E^{c} \cap\left(a, \lambda_{n}\right) \\ 0, & \text { elsewhere. }\end{cases}
$$

From Lemma 1 it follows that $\exp \left(i H \theta_{j}^{n}\right)$ belongs locally to $L^{p}(\mathbf{R})$, for $j=1,2, \ldots$. The only point where this is not clear is at $\lambda_{n}$, however, in a neighborhood of $\lambda_{n}$ one can write $\theta_{j}^{n}=v+w$; where,

$$
v(t)= \begin{cases}\pi-\alpha / 2, & t \in E^{c}, t \leqslant \lambda_{n}, \\ 0, & t \in E, t \leqslant \lambda_{n} \text { or } t \in E^{c}, t>\lambda_{n}, \\ \alpha / 2, & t \in E, t>\lambda_{n},\end{cases}
$$

and

$$
w(t)= \begin{cases}\alpha / 2, & t \leqslant \lambda_{n}, \\ 0, & t>\lambda_{n} .\end{cases}
$$

Lemma 1 can be applied to this decomposition to show that $\exp \left(i H \theta_{j}^{n}\right)$ is in $L^{p}$ in a neighborhood of $\lambda_{n}$, for $j=1,2, \ldots$.

It follows from Lemma 2 that the functions

$$
\Theta_{j}^{n}(z)=\exp \left[\pi^{-1} \int \theta_{j}^{n}(t)(t-z)^{-1} d t\right]-1
$$

belong to $H^{p}$, for $j=1,2, \ldots$.

Writing out equation (5) for the functions $\left\{\left(\Theta_{j}^{n}\right)^{+}\right\}_{j=1}^{\infty}$ one obtains that for a.e. $x \in E$ 
(6) $\left[\left(H_{E}-i \mu\right) \exp \left(i H \theta_{j}^{n}\right)\right](x)=i \sqrt{\mu^{2}+1}\left[1+\frac{1}{\pi} \int_{a-1 / j}^{a} \frac{\exp \left(i H \theta_{j}^{n}\right)(t)}{t-x} d t\right]$.

The next step is (for $n$ fixed) to take the limit as $j \rightarrow \infty$. It is clear that on $E$ the sequence $\exp i\left[H \theta_{j}^{n}\right]$ converges uniformly to $\exp \left(i H \theta_{n}\right)$, where

$$
\theta_{n}(t)= \begin{cases}\alpha / 2, & t \in E \\ \pi, & t \in E^{c} \cap\left(a, \lambda_{n}\right), \\ 0, & \text { elsewhere. }\end{cases}
$$

The limiting behavior of the integral on the right side of equation (6) requires some discussion.

Let $\theta_{b}^{n}=\chi_{(b, \infty)} \theta_{j}^{n}$ (recall $\left.a<b<m\right)$, note that $\theta_{b}^{n}$ is independent of $j$. Also on the interval $(a-1 / j, a)$

$$
\exp \left(i H \theta_{j}^{n}\right)(t)=\frac{(b-t) \exp \left(i H \theta_{b}^{n}\right)(t)}{\sqrt{a-t} \sqrt{t-a+1 / j}}
$$

Moreover,

$$
\frac{1}{\pi} \int_{a-1 / j}^{a} \frac{d t}{\sqrt{a-t} \sqrt{t-a+1 / j}}=1, \quad j=1,2, \ldots,
$$

and the function $f_{n}(t)=(b-t) \exp \left(i H \theta_{b}^{n}\right)(t)$ is continuous at $t=a$. It follows from routine considerations of approximate identities that

$$
\lim _{j \rightarrow \infty} \frac{1}{\pi} \int_{a-1 / j}^{a} \frac{\exp \left[i H \theta_{j}^{n}\right](t)}{t-x} d t=f_{n}(a)(a-x)^{-1}, \quad x \in E .
$$

This leads to the identity

$$
\left(H_{E}-i \mu\right) k_{n}=i \sqrt{\mu^{2}+1}\left[f_{n}(a) \sigma_{a}+1\right] ;
$$

where, the function $k_{n}=\exp \left(i H \theta_{n}\right)$ is in $L^{p}(E)$ and $\sigma_{a}(x)=(a-x)^{-1}$.

Now it is clear that the functions

$$
g_{n}=\left(k_{n}-k_{1}\right) /\left(f_{n}(a)-f_{1}(a)\right)
$$

satisfy

$$
\left[H_{E}-i \mu\right] g_{n}=i \sqrt{\mu^{2}+1 \sigma_{a}}, \quad n \geqslant 2 \text {. }
$$

LEMma 3. The functions $\left\{g_{n}\right\}_{n=2}^{\infty}$ defined by (8) are linearly independent in $L^{p}(E)$.

Proof. Suppose $\Sigma_{k=2}^{N} a_{k} g_{k}=0$ on $E$ with $a_{N} \neq 0$. Multiplying by $\exp \left(-i H \theta_{1}\right)$ one obtains $\Sigma_{k=2}^{N} a_{k} \phi_{k}=0$ on $E$, where 


$$
\phi_{k}=\left(f_{k}(a)-f_{1}(a)\right)^{-1}\left[\exp \left[ \pm \pi i H \chi_{\left(E^{c} \cap \cup_{j=2}^{k} I_{j}\right)}\right] ;\right.
$$

here, the plus sign is chosen if $\lambda_{n} \uparrow$ and the minus sign, if $\lambda_{n} \downarrow$. There are subsets of $I_{N} \cap E$ on which $\phi_{N}$ is unbounded and $\phi_{2}, \ldots, \phi_{N-1}$ are bounded (see the remarks at the end of $\S 1$ ). It is clear that $a_{N}$ must be zero. This contradiction completes the proof.

The preceding lemma along with the fact that the functions defined by (8) satisfy (9) imply for $\lambda \in R_{p}, \lambda$ purely imaginary, the operator $H_{E}-\lambda$ has an infinite dimensional null space. This result is contained in the following:

THEOREM 1. Let $E$ be a bounded measurable subset of the real line which is not equal a.e. to a finite union of intervals. . Let $p$ satisfy $1<p<2$ and set

$$
R_{p}=\left\{\lambda \in \mathbf{C}: 2 \pi q^{-1}<\arg [(\lambda+1) /(\lambda-1)]<2 \pi p^{-1}\right\} .
$$

Then for each $\lambda \in R_{p}$ the operator $H_{E}-\lambda I$ has an infinite dimensional null space.

Proof. The above work shows that for every $\lambda \in R_{p}$ which is purely imaginary the null space of $H_{E}-\lambda I$ is infinite dimensional. Moreover, the functions $h_{n}=g_{n}-g_{2}$ for $n>2$; where the function $g_{n}$ is defined by (8) is a linearly independent solution set of the equation $\left[H_{E}-i \mu\right] \phi=0$. If one replaces the $\alpha$ in the definition of $\theta_{n}$ by $\log [(\lambda+1) /(\lambda-1)] / 2 i$, then the $h_{n}$ 's corresponding to $\lambda$ in $R_{p}$ are seen to be $L^{p}(E)$ valued functions which depend analytically on $\lambda$ in $R_{p}$. This completes the proof.

3. Concluding remarks. Recall that a linear operator $T$ on a Banach space $X$ is called Fredholm in case $T X$ is closed on both $T$ and its adjoint $T^{*}$ have finite dimensional kernels. The essential spectrum of $T$ is defined to be the set of all complex $\lambda$ such that $T-\lambda I$ is not Fredholm.

Theorem 1 implies that in the case where $E$ is not a finite union of intervals, then the set $R_{p}$ belongs to the essential spectrum of $H_{E}$ on $L^{p}(E)$, for any $p>1$. This result, for $p>2$, follows from Theorem 1 by duality.

Theorem 1 does not rule out the possibility that the range of $H_{E}$ on $L^{p}(E)$, $p \neq 2$, is always closed. It would be interesting to know the answer to this question as well as the results analogous to Theorem 1 for the reduced Hilbert transforms.

Added In Proof. There are two papers by V. D. Frolov (Mat. Issled. 1 (1971), 146-157; Mat. Issled. 32 (1974), 172-181) that deal with singular integral equations on an infinite system of arcs.

\section{REFERENCES}

1. K. F. Clancey, Completely seminormal operators with boundary eigenvalues, Trans. Amer. Math. Soc. 182 (1973), 133-143. 
2. A. M. Garsia, Topics in almost everywhere convergence, Lectures in Advanced Math., vol. 4, Markham, Chicago, III., 1970. MR 41 \#5869.

3. I. C. Gohberg and N. Ja. Krupnik, Singular integral operators with piecewise continuous coefficients and their symbols, Izv. Akad. Nauk SSSR Ser. Mat. 35 (1971), 940-964 = Math. USSR Izv. 5 (1971), 955-979. MR 45 \#981.

4. R. K. Juberg, Finite Hilbert transforsm in $L^{p}$, Bull. Amer. Math. Soc. 78 (1972), 435-438. MR 45 \#982.

5. E. Shamir, Reduced Hilbert transforms and singular integral equations, J. Analyse Math. 12 (1964), 277-305. MR 29 \#2617.

6. E. C. Titchmarsh, Introduction to the theory of Fourier integrals, Clarendon Press, Oxford, 1937.

7. F. Tricomi, Integral equations, Interscience, New York, 1957. MR 20 \#1177.

8. H. Widom, Singular integral equations in $L_{p}$, Trans. Amer. Math. Soc. 97 (1960), 131-160. MR 22 \#9830.

9. A. Zygmund, Intégrales singulières, Lecture Notes in Math., vol. 204, Springer-Verlag, New York, 1971.

10. - Trigonometrical series. Vols. I, II, 2nd rev. ed., Cambridge Univ. Press, New York, 1959. MR 21 \#6498.

DEPARTMENT OF MATHEMATICS, UNIVERSITY OF GEORGIA, ATHENS, GEORGIA 30602 